\title{
TEACHING GENETICS WITH AN ELECTRONIC COMPUTER
}

\author{
J. L. CROSBY \\ Botany Department, Durham Colleges in the University of Durham
}

\section{INTRODUCTION}

Received 6.iii.6 $\mathrm{I}$

MATERIAL for practical classes in genetics has always presented a difficult problem, which is aggravated by the fact that the best times of the year for practical plant genetics are occupied by vacations or examinations. Cytology can be dealt with fairly easily; suitable material can usually be provided all the year round or can be fixed and kept until required, and the quantities needed are small.

The biggest dificulty in planning genetical work arises from the time that must elapse between making the crosses and scoring the progeny, and this becomes much worse when, as in plant genetics, the experimental material cannot be made to conform to a university timetable. Animals provide better opportunities, though the use of vertebrates such as mice depends on having animal house facilities. By far the best and most widely used organism is Drosophila melanogaster, but even this has its limitations, and its extensive use in genetics courses may prove wearisome to all but the keenest students. Nor can it always adequately illustrate the points that one might wish.

Recourse may be had to numerical examples (e.g. published results of other people's experiments), to concoctions of loose peas or maize grains, or to entire maize cobs. The first are unconvincing, as being selected to give the right answer, the second are obvious fakes, and maize cobs which have been counted by many generations of students are rather dull. Nor do any of these allow the students either to make or even witness the crosses. By far the most effective data, because they hold the interest of the students, are those from experiments in which they themselves have taken part and whose results are not predetermined - the possibility of the unexpected is always attractive.

Evolution experiments, by their nature, present the greatest difficulties. They may be carried on over a number of years by successive classes, or by using Drosophila within the compass of a university course. The latter, however, involves either too much scoring for the class, or too much for the teacher.

Attempts have been made to produce mechanical models of evolutionary systems, but the analogies are far from perfect, and time remains a drastically limiting factor.

Some of the difficulties can be overcome where the course is a specialist one in genetics, and more time and better facilities may be available. In writing this paper, I have particularly in mind the 
situation where genetics is being taught as part of a course in botany or zoology.

In February r 960 the final honours classes in botany and zoology at Durham were introduced to a new "organism". As Fraser (r957) pointed out, the form of the number in an electronic computer allows rather precise analogies with genetic systems. Genetic models may be formed of electronic organisms on which can be impressed whatever rules of genetics one chooses; great flexibility of the system allows the exemplification of a much wider range of genetic phenomena than could easily be achieved with more orthodox material, and interfering side-effects can be eliminated or deliberately included as desired. The superimposing of quasi-random variation brings the systems much closer to reality, and eliminates any question of the predetermination of results.

One thinks primarily of the electronic computer as a very fast calculating machine; but its ability is by no means restricted to calculation. The complex arithmetic which it can perform requires a great deal of administration, and it is the administrative activities of the machine which form the basis of its use in genetic simulation.

To use the machine purely to perform computations from genetical or evolutionary equations would be pointless, and it must be emphasised that the experiments described in this paper are in no sense based on such calculations. Instead, pseudo-organisms are created within the computer, and provided with genotypes which can have phenotypic expression. They are allowed to reproduce according to such laws of chance and genetics as may be desired. They are admirable subjects for experiment.

\section{PRINCIPLES OF GENETIC SIMULATION}

In the accounts which follow, the aim has been to explain the basis of the electronic analogies which have been used, so that the reader may judge their validity for himself; no special account of computing techniques is given.

The experiments were carried out with a Ferranti "Pegasus" computer, but the general principles have a wider applicability. The working units ("words") of this computer are linear strings of $3^{8}$ digits each of which may take one of two values, o or I. Ordinarily, this string of digits is used in one of two ways-either as a simple code giving instructions (" orders") to the machine, or as a number on a binary scale. But it can in fact be used for any purpose for which a string of digits is suitable, and such a string can be manipulated in several ways - as freight trains may be broken down and reorganised in a marshalling yard. Several words may be shuffled, and their contents recombined. Single digits or groups of digits may be treated in isolation, and may be used to determine activities of the machine; such activities may be regarded as phenotypic expressions of the digits. 
In short, the word of the computer provides us with a simple but excellent analogy with a genetic system. The two forms, o or I, of each digit may be considered as two alleles of a gene; these may be given phenotypic expression by causing the precise activity of the machine to depend on the value of the digit at a particular locus in the word. For example, the machine could be instructed to print "round" if the digit is o, or "wrinkled" if it is I. Among other things, we may introduce analogies of complementary gene action, additive effects, inhibition, and incomplete penetrance, by making the phenotypic expression of a particular digit depend on the value of the digits (i.e. the allelic state) at other loci, or by allowing it to be affected by chance.

One important principle is that the results of computer experiments must be no more predictable than those from experiments with living organisms; there must be no question of predetermination of results by the teacher. The appropriate element of unpredictability may be introduced by the use of sequences of pseudo-random numbers calculated by the machine. Such sequences can never, of course, be truly random, but by the use of suitable generating equations it is possible to produce sequences which in their important properties closely simulate randomness, and in practice may be safely treated as though they were truly random. For convenience, the term "random" will be used throughout this paper for these pseudorandom processes. The sequence used in the programmes to be described is the same as that of an earlier paper (Crosby, I96o), and is a cycle of over two thousand million integers; the cycle may be started at any of its integers, and by allowing the class to choose the starting number the possibility of any predetermination by the teacher is ruled out. Indeed, unless a record of the starting number is kept, the results of any experiment cannot be deliberately repeated.

\section{MEIOSIS AND CROSSING-OVER}

In the simplest analogy, the word may be considered as a single chromosome or chromatid with a centromere and 37 gene loci, each gene having two possible alleles. The versatility of the computer allows us to do many things with such a chromosome, some of them closely analogous to natural processes.

Fig. I shows four such words arranged as two pairs; we are going to consider these as two homologous (but allelically different) chromosomes during meiosis, at the transition from pachytene to diplotene; it is supposed that the chromosomes have divided into chromatids but chiasmata have not yet formed. One digit is the centromere and is printed as an asterisk; teleprinter limitations require this to appear divided too, but this limitation is readily appreciated by the class and causes no confusion.

Now it is quite a simple matter to make the computer exchange sections of words; for example, it could be instructed to transpose the 
twelve left-hand digits of the two central words; this would represent chiasma formation with, of course, genetical crossing-over. We could then regard the four words as the four daughter chromosomes of anaphase II. If we had wished, we could have allowed the possibility of more than one chiasma forming at diplotene.

In fig. 2, the four words represent the daughter chromosomes at anaphase II after two chiasmata. Numbering from the top and from

I I IIIIIIIIIIIIIIIIIIIIIIIIIIIII I I

I I I I I IIIIIIIIIIIIIIIIIIIIIIIII * I I $0000000000000000000000000000000000 * 000$ $0000000000000000000000000000000000 * 000$

FIG. 1.-Four words of the computer, representing paired homologous chromosomes each divided into two chromatids, at the transition from pachytene to diplotene but before chiasma formation.

the left, one chiasma involved chromatids I and 4 between the 9 th and roth digits, and the other chromatids 2 and 4 between the 2oth and 2 Ist digits (the reader may disagree with this interpretation; I fell into a similar trap when programming this experiment).

This may be demonstrated to the class on a cathode-ray monitoring tube, and printed out by the machine. Fig. 2 is a facsimile of such a print-out, which suggests a useful exercise for the class. Students are

000000000 I I I I I I I I I I I I I I I I I I I I I I I I I * I I I

I I I I I I I I I 00000000000I I I I I I I I I I I I I I* I I I

$0000000000000000000000000000000000 \% 000$

I I I I I I I I I I I I I I I I I I I $100000000000000 \% 000$

Fig. 2.-Four words of the computer, representing four homologous daughter chromosomes at anaphase II, after two chiasmata.

required to make diplotene diagrams corresponding to print-outs of anaphase II, and find that it isn't always as obvious as it looks; this has the merit of making them think carefully about the logical consequences of chiasma formation.

Chiasma position must not be determined by the teacher; it is one of the variables of the experiment and therefore unpredictable. The simplest way of applying randomness to chiasma formation would be as follows. There are 37 possible positions in which a chiasma may form, and one of these may be chosen by generating a random number in the range I to 37 ; two non-sister chromatids would then be chosen at random and their genes (digits) distal to the chosen position transposed. A second chiasma could be formed in the same way; the two chiasmata cannot be formed simultaneously, and it is essential that the more distal one be done first.

However, in this simple form the process would not give a very realistic analogy. Chiasma formation is not random; it is well known 
that as a general rule chiasmata tend to occur less frequently near the centromere and the ends of the chromosomes. What has in fact been done is to take account of end and centromere interference by arranging that the randomisation process will most readily pick positions away from ends and centromere, and least readily close to them. This is done by storing with different frequencies the numbers I to 37 in $5^{12}$ locations in the computer, and choosing one of the locations at random. The frequencies of the numbers follow roughly a flattened normal distribution curve, and the numbers representing the more central positions are therefore the most frequent; thus the chiasma position will most commonly be in the central region of the chromosome.

Chiasma interference has also been taken into account, in the following way. Initially, two chiasma positions are chosen for each meiosis. If they coincide or are adjacent, only the first one chosen is allowed to stand and there will only be a single chiasma. If they are well separated, both are allowed to stand and we have double chiasmata. When the two positions are fairly close together, a random process is introduced which increases the chance of the second chiasma being allowed to stand the farther it is from the first. By the way in which this has been done, chiasma interference is greatest near the ends and centromere.

Obviously, any other pattern of chiasma localisation could have been chosen, and any degree of chiasma interference. The choice was quite arbitrary, and was designed to give the most suitable results for teaching purposes. It would have been easy to introduce chromatid interference, but this has not been done; nor have more than two chiasmata been allowed.

\section{LINKAGE AND CHROMOSOME MAPPING}

The visual demonstration of chiasma formation in the monitoring tube makes an excellent introduction for the class, and leads naturally into the experiment which is developed from it. This is the construction of a chromosome map from a series of 3-point linkage tests scored by the students themselves.

Eleven of the digits on the chromosome we have been considering have been chosen as gene loci in this experiment. Each gene is named in the orthodox manner, and its phenotype is a symbol which is printed as a single character. For each gene, I is taken to be the recessive allele and causes the appropriate character to be printed; $o$ is the normal dominant and always causes + to be printed. The class has no prior knowledge of the positions or order of the genes on the chromosome.

Each test-cross is performed, with complete coupling, as follows. Meiosis proceeds (from fig. I) to anaphase II. One of the four daughter chromosomes is then chosen at random to be one of the 
chromosomes in a single test-cross offspring; the homologous chromosome from the testing parent is of course always triple recessive. Thus, as in any test-cross, the phenotype of the offspring is the same as the genotype of the chosen chromosome; the characters of the phenotype are then printed from the chosen chromosome for each of the three loci under test-the appropriate symbol if the digit is the recessive $\mathrm{I}$, and + if it is $\mathrm{o}$. The order of printing is not necessarily that in which the genes occur on the chromosome, since this is one of the things which have to be determined by the student; the order adopted is alphabetical according to the gene names. As an example of this process, we may consider fig. 2 and a test-cross with the genes star *, dash - , and tipsy /. From the left, these are in digit positions 4,12 and 33 respectively, indicated by dots under the diagram. Had the bottom chromosome of this figure been chosen, the offspring would have appeared as $-*+$ since on that chromosome the digits involved are I, I and o respectively.

This process may be repeated from the position of fig. I as often as required, using successive (and therefore different) numbers from the randomisation sequence. In this way, we can produce a family of as many individuals as desired; a convenient size is 200, and this takes about $\mathrm{I} \frac{1}{2}$ minutes to produce. One student is given the print-out sheet of one family and asked to score it, and to determine the order of the genes and the percentages of recombination between them. Fig. 3 is a facsimile of such a print-out, and the reader is invited to score it for himself, and draw the appropriate conclusions before looking at the map which follows later. Although the three genes of this family were chosen deliberately for this paper, the family itself is the only one produced for these three, and is therefore not a selected example.

Subsequent test-crosses, with other gene combinations, start from different numbers in the random sequence and thus have quite different patterns of chiasma formation and chromosome selection.

There are several ways in which the full experiment may be carried out. The teacher may predetermine the test-crosses to be done, and allot them to members of the class; this has the advantage that the crosses may be chosen not only to allow mapping, but also to produce good teaching points, such as the shortage of double cross-overs due to chiasma interference, and the underestimation of long distances due to double crossing-over. On the other hand, there is a considerable advantage, from the point of view of class interest, in allowing the students to choose the crosses for themselves; but particularly with a small class their choice may be such that it may not allow unambiguous determination of the gene order. Where the computer is readily accessible and repeated short visits can easily be made, the most realistic method may be used; the students decide on a few exploratory test-crosses, and from the results of these can plan subsequent series of crosses to give an accurate map of the genes. 


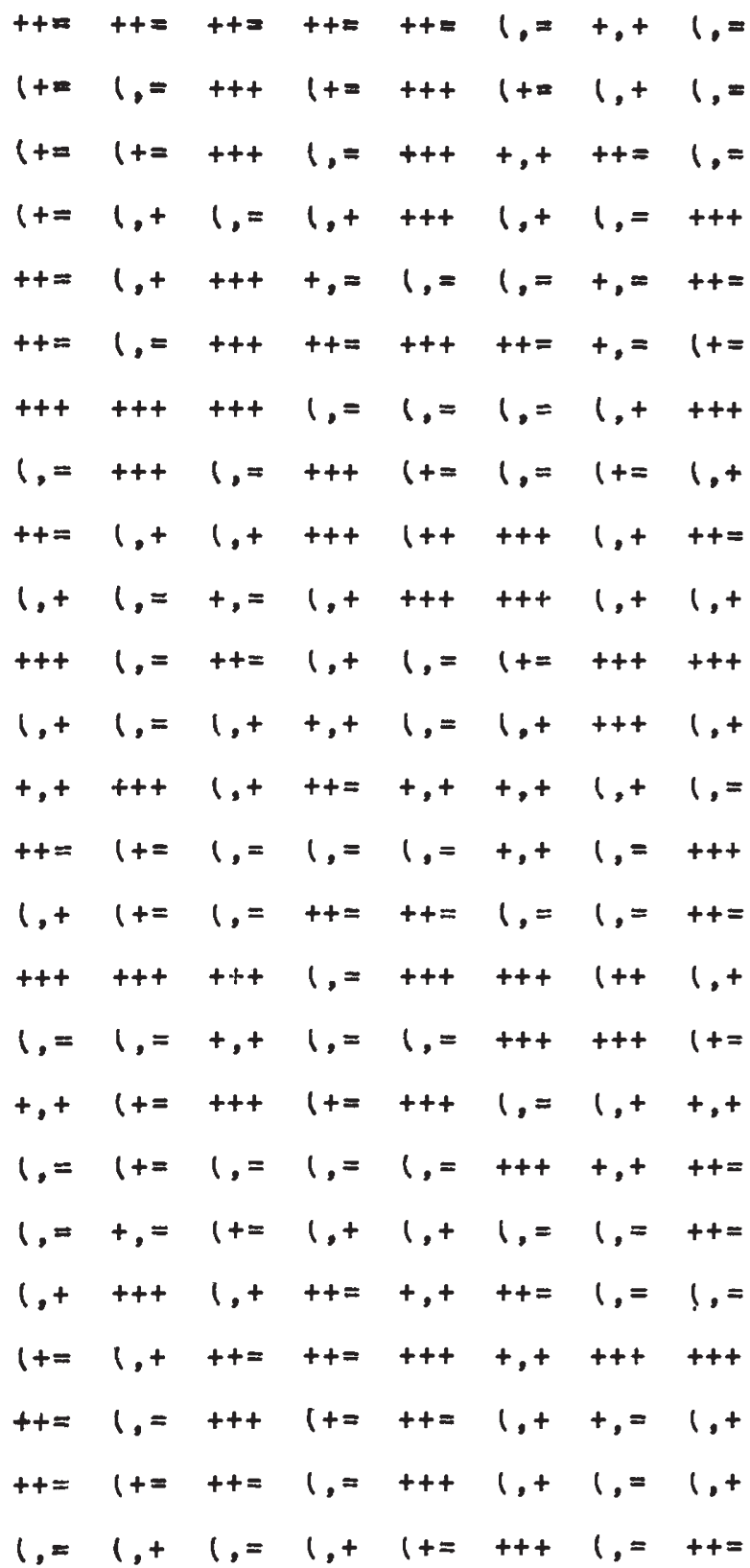

Frg. 3.-Facsimile of a family from a 3-point test-cross. The genes involved are arc curly mimic with phenotypes $(,=$ respectively. The dominant phenotype in each case is + . The tested parent had +++ on one chromosome and $(,=$ on the other, though not necessarily in that order. The reader is invited to score this family for himself. 
Although the Durham University computer is situated 16 miles away in Newcastle, and so is not easily accessible to Durham classes, a method similar to that last described is quite convenient because the classes involved are in any case too large to handle in a single visit to the computer. First, half the class visits the computer, choosing which test-crosses they will perform subject to the limitation that all genes must be covered by their choice. The test-cross families are scored by the students involved, and from the results a provisional map of the eleven genes is constructed by an assembly of the whole class. The second half of the class then decides which are the best test-crosses to complete an accurate map, and allot these among themselves; their trip to the computer leads to a final mapping of the chromosome during a further assembly of the whole class.

The way in which the final map is determined will depend upon the standing of the class and the intentions of the teacher. With an advanced class, refined mathematical methods may be used, for example to allow corrections for interference; indeed, computer results form an excellent basis on which such mathematical techniques may be taught. At Durham, this experiment is performed at a fairly elementary stage, where such a treatment would be inappropriate, and the primary aim is to help the class to understand the general principles of chromosome mapping based on test-crosses; the mapping is straightforward, if somewhat approximate.

When the class has completed the genetical map, they are then shown the "cytological " map, i.e. a diagram giving the exact digital positions of the eleven genes. Fig. 4 shows the first genetic chromosome map produced by our second-year class, together with the cytological map for comparison. The resemblance to the familiar Drosophila maps is striking, and the essential difference between cytological and genetical maps is clearly brought home to the class. The figure shows the well-known interference effect by which genes in the centre of the chromosome appear to be relatively farther apart than they really are, while those near the ends and the centromere appear much closer together.

The effect of double cross-overs in causing underestimation of map distances was shown in several test-crosses. For example, the following distances were calculated from individual crosses (in each case the third gene of the cross did not lie between the given two): $d-t$, two separate test-crosses gave $47 \mathrm{cM}$ and $46.5 \mathrm{cM} ; b-a, 36 \mathrm{cM}$. The distances as finally determined in this experiment were $56.6 \mathrm{cM}$ and $4^{\mathrm{I}} \cdot 9 \mathrm{cM}$ respectively.

Chiasma interference will have been observed by those readers who have dealt with fig. 3 ; it was also well illustrated by several of the crosses involved in the production of the map in fig. 4. For instance, in one family of 200 there were 36 single cross-overs in one region and 64 in the other, but only 8 double cross-overs instead of the expected $16\left(\chi_{[1]}^{2}=7 \cdot 8\right)$. 
These of course are points which could be illustrated with Drosophila, although a series of test-crosses involving eleven easily scorable linked genes would be beyond the resources of most departments.

There is, however, one instructive experiment which could not be made with living material. A series of meiosis print-outs is obtained

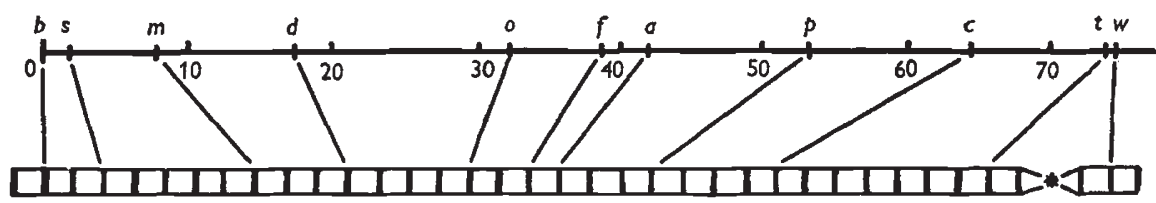

FrG. 4,-Comparison of genetic and cytological maps. The upper map is that determined from a series of test-crosses scored by the class. The lower map is the "cytological" one and shows the actual digital positions of the eleven genes.

as first described. A test-cross is then run, starting from the same number in the random sequence as the print-outs; the test-cross repeats the same sequence of random numbers, and the individuals of the test-cross family therefore correspond, in order, to the previously printed meioses. So when the precise digital positions of the genes

IIIIIIIIIIIIIIIIIIIIIIIIIIIIIIIIII*III $00000000000000000 I I I I I I I I I I I I I I I I I$ III IIIIIIIIIIIIIIIIII00000000000000000*000 $0000000000000000000000000000000000 * 000$
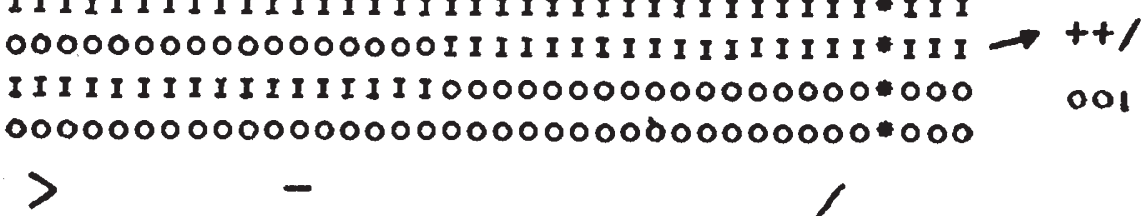

IIIIIIIIIIIIIIIIIIIIIIIIIIIIIIIIII*III

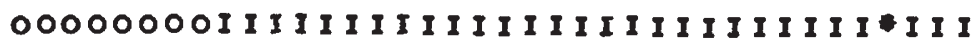
IIIIIIII $00000000000000000000000000 * 000$ $0000000000000000000000000000000000 * 000$ 001

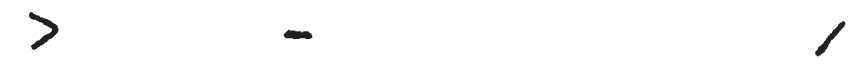

\section{IIIIIIIIIIIIIII0000000IIIIIIIIIIII*III 000000000000000 IIIIIIIIIIIIIIIIII*III IIIIIIIIIIIIIIIIIIIIIII000000000000*000 $0000000000000000000000000000000000 * 000$}

FIG. 5.-Print-outs of anaphase II with the corresponding test-cross progeny shown on the right. For full explanation see text. The digital positions of the genes are indicated ; each phenotype has its digital equivalent shown below it. The lowest example shows a genetically undetectable double crossover.

are known, it is possible for the members of the class to tell which chromosome from each printed set of anaphase II was chosen to produce the corresponding individual in the test-cross family. This is illustrated in fig. 5, which is taken from an actual teaching run. This figure again is a facsimile, the corresponding phenotypes having been excised from the family print-out. The lowest example provides a particularly neat teaching point. It will be seen that the individual 
produced was a triple recessive non-crossover; but there is no noncrossover recessive chromosome. Closer examination shows that the chromosome involved must have been the top one, which has a double crossover between dash (-) and tipsy (/); this provided a good cytological demonstration of a genetically undetectable double crossover between two rather distant genes, and reinforces the point of underestimation of long distances when determined directly. The example comes, in fact, from one of the two families involving $d$ and $t$ cited above.

The ability to allow examination both of a meiosis and of the offspring derived from it is one advantage which electronic organisms can claim over living ones.

\section{POPULATION GENETICS}

The possible range of genetic analogy may be greatly extended by using other methods of organising the genetic material within the computer. We are not restricted to the interpretation of a word as a series of linked loci; it could easily be made to represent 38 unlinked genes, or we could consider pairs of digits as allelic pairs of such genes.

Suppose we wish to deal with three unlinked genes; $A / a, B / b$ and $C / c$. We could represent the genotype of a diploid organism by using six digits in three pairs, each representing one allelic pair. If o is the dominant and I the recessive, then Iooro I would represent $a A B b C c$ or, more conventionally, $A a B b C c$. Let us consider the production of an $F_{2}$ by self-fertilisation of such a triple heterozygote.

Since the genes are unlinked, we do not need to take chiasma formation into account, and our electronic meiosis can be simplified. By deciding on simple adherence to Mendel's second law, it follows that to produce a gamete we have to pick at random one digit from each of the three pairs. Suppose for example that random choice falls on digits I, 3 and 6 (from the left), that is ro r representing a gamete $a B c$. We store these in the odd positions in a new word which is to represent the offspring, thus, I-O-I-. A second random choice from the parent, one from each pair as before, gives us the second gamete; fertilisation occurs when these digits are placed in the even positions and we then have the new individual. Thus, if the second choice was digits I, 4 and 5 , the gamete would be I Io $(a b C)$ and the zygote I Iо I $(a a B b c C)$. Our electronic organism has reproduced itself once by self-fertilisation.

We may now cause the machine to print the new genotype, or to take account of dominance and print the phenotype, preferably not as gene letters but as symbols representing the characters of the organism, as in the crossing-over experiment. This whole process may be repeated to give as large a family as desired. The print-out is given to the class to be scored, as a family of living organisms would be. It would be easy for the scoring and counting to be done by the 
machine, but this would destroy the object of making these experiments as close in form to the real thing as possible.

Different kinds of cross- or self-fertilisation may be simulated in this way, and it is only a short step from controlled crosses to the simulation of breeding populations which may be followed over a series of generations. This gives the possibility of conducting evolutionary experiments. Any desired degree of inbreeding or outbreeding may be imposed on such a population, either in general terms or by detailed simulation of particular kinds of breeding system. Both natural and artificial selection can easily be introduced. Once again, the use of a randomisation technique is fundamental to these experiments.

If one word is taken to represent an organism, many hundreds can be kept within the computer; there is, however, a practical limit to population size, irrespective of the capacity of the machine. In one important respect electronic analogy breaks down-real organisms can reproduce simultaneously, electronic ones can only do so one at a time. This means that if the population size is doubled, the time taken for the population to reproduce itself is doubled. A balance has therefore to be struck between the small populations which would show an excessive Sewall Wright effect, and large populations which would either take too much time for class experiments or would give too few generations. Running time for these programmes is always an important consideration.

In the experiments which are to be described, population size is kept constant during each evolutionary run (though variable between runs), the electronic organisms are hermaphrodite and diploid and behave in the same way as annual plants with no overlapping of generations, and the outbreeding systems are panmictic. These are purely restrictions of convenience, and it would be easy to arrange otherwise if the extra time required by the more complex programmes was relatively unimportant.

When the size and the starting genetic constitution of the population have been decided (that is, the gene frequencies, heterozygosity conditions etc.), the required number of organisms are stored as successive words of the computer, the frequencies of the various genotypes conforming to the required initial conditions. The population is then allowed to reproduce itself as follows.

A parent is chosen at random, and a gamete extracted as previously explained. If the population is panmictic, a second parent is chosen at random and the second gamete extracted from it. The two gametes are combined and the resulting new individual is stored in another part of the computer. This process is repeated until the number of offspring equals the population size, and the whole new generation is transferred to replace the old one. We can now start to produce a further generation from the new population, and so on. It will be noticed that during any generation some organisms will by chance 
reproduce several times, some once only, and some not at all, as in any ordinary population.

A simple modification allows for complete inbreeding-only one parent is selected for each reproduction, and provides both gametes. It is easy to introduce any intermediate degree of inbreeding, predetermined as a fraction. When for each offspring one parent has been chosen, this fraction is referred to a random number; this process determines whether the same parent shall be used for the second gamete or a new choice made, the probability of the former event being equal to the inbreeding fraction.

If the experiment is being performed purely as a demonstration, the genetic constitution of the population may be analysed by the computer and printed out as desired. But if it is intended as an experiment for class participation, then it may be arranged to resemble more closely an experiment with living organisms, so that the work done by the students is as far as possible analogous to more orthodox genetical techniques. One example of each of these approaches, as carried out at Durham, will now be given in order to illustrate in a simple way some of the potentialities of evolutionary experiments by computer.

\section{THE SEWALL WRIGHT EFFECT}

Computer analogy provides an excellent way of showing random fluctuation in gene frequency in small populations. This experiment is more suitable for demonstration than for student participation, which would increase the amount of time required very considerably. One consequence of the fluctuation being random is that the time which is likely to elapse before anything interesting happens is quite unpredictable - a gene may become fixed in a few generations, or be nowhere near fixation after very many; one must be prepared for long runs. In its original form, this experiment involved two unlinked genes; this number has been increased to eight, giving a greater likelihood of at least one gene becoming fixed in a reasonable time. Increase in gene number has not added to the running time-a simple method of gamete selection (Fraser, I 960) can deal with many genes simultaneously.

Population size can be varied from 16 to $5^{12}$, but the larger sizes are not practicable unless ample time is available; at a size of 64 , one generation takes 7 seconds. The population is fully outbreeding, and there is no selection. All genes start with 50 per cent. frequency, and any subsequent fluctuations are due purely to chance. Gene frequency can be followed on a monitoring tube, but this involves interpretation of binary numbers and may be difficult except near zero (extinction of one allele). It is easier for the class to follow gene numbers as they are printed at the completion of each generation. They find this quite exciting, especially if each student is allotted a particular gene, and it brings home to them forcibly the amount of fluctuation in gene frequency that may be caused purely by chance. 
To illustrate the kind of results that may be obtained from this experiment, fig. 6 shows the first 120 generations from the first teaching run with the 8-gene programme, in a population of 64 individuals. To minimise confusion, only four of the eight genes are considered in this figure, the choice being governed by considerations of clarity; two of them are seen to have become fixed during the period covered by this figure. Of the other four genes, one became fixed after 63 generations and another at 16o. After 199 generations four of the eight genes were far from fixation, although two of them had at one

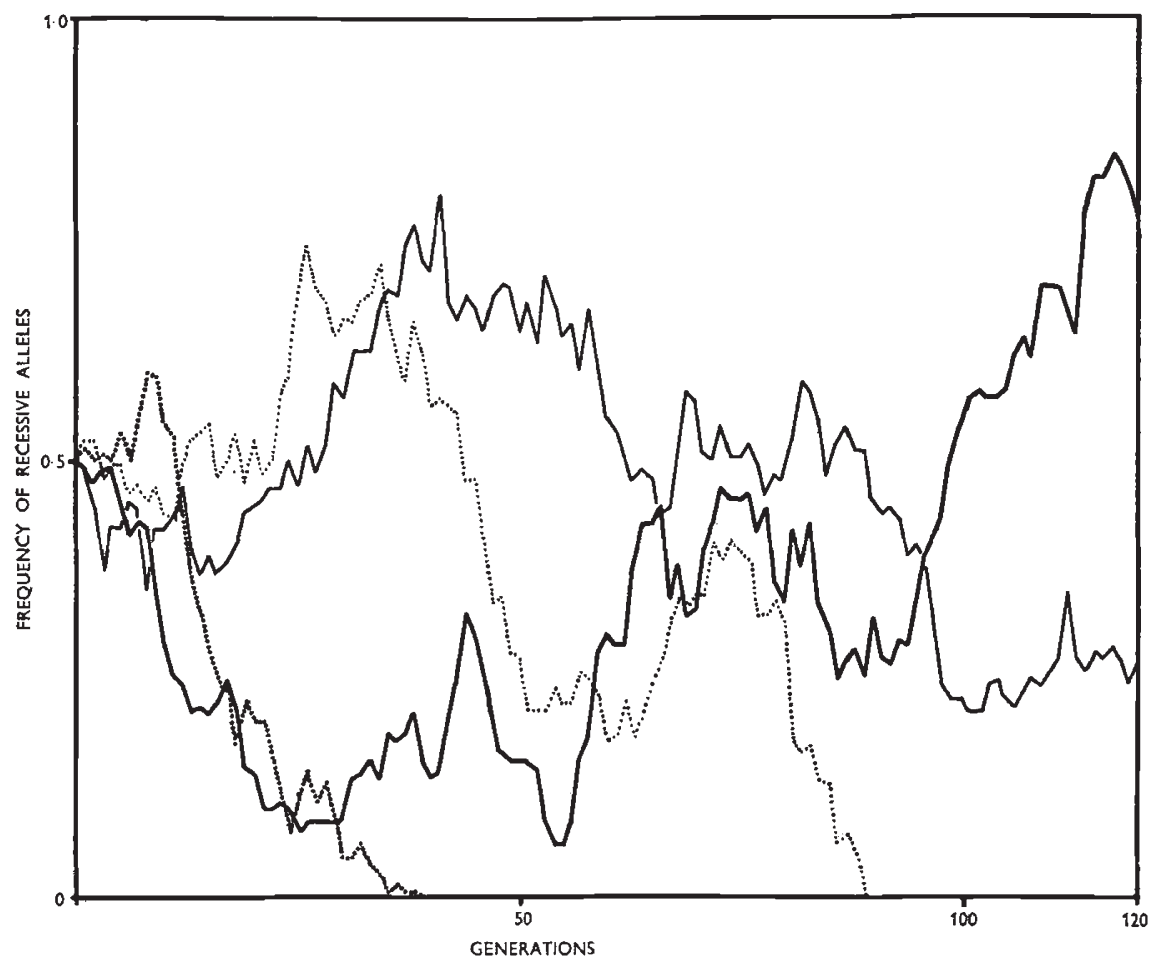

FIG. 6.-The Sewall Wright effect. The frequencies of the recessive alleles of four unlinked genes during the first 120 generations of a population of 64 individuals, with no selection. Changes in gene frequency are due entirely to chance.

time been very close to it. During the same session, a run with a population of size 16 saw seven of the eight genes fixed within 13, I6, 20, 24, 52, 55 and $5^{6}$ generations respectively.

The class is able to compare populations of different sizes, but the comparisons are themselves affected by chance. The students of subsequent years will be able to get a clearer picture, because the results of their predecessors will be available to them; freak results will be welcome then, when they can be seen in perspective, but in the early stages they are liable to be embarrassing, although they do help to convince the class that the whole thing is not fixed by the teacher. 


\section{INBREEDING AND OUTBREEDING}

This programme is designed to illustrate the important differences between inbreeding and outbreeding in respect of the degree of heterozygosity, the genetic variability of populations, and response to selection. The populations may be panmictic or fully inbreeding, or of any desired intermediate degree of inbreeding.

Unfortunately, in order to give an adequate number of generations in a reasonable time, it has been found necessary to restrict the population size to 256 , and the Sewall Wright effect is sometimes quite marked. Even with this size, a generation takes about 50 seconds;

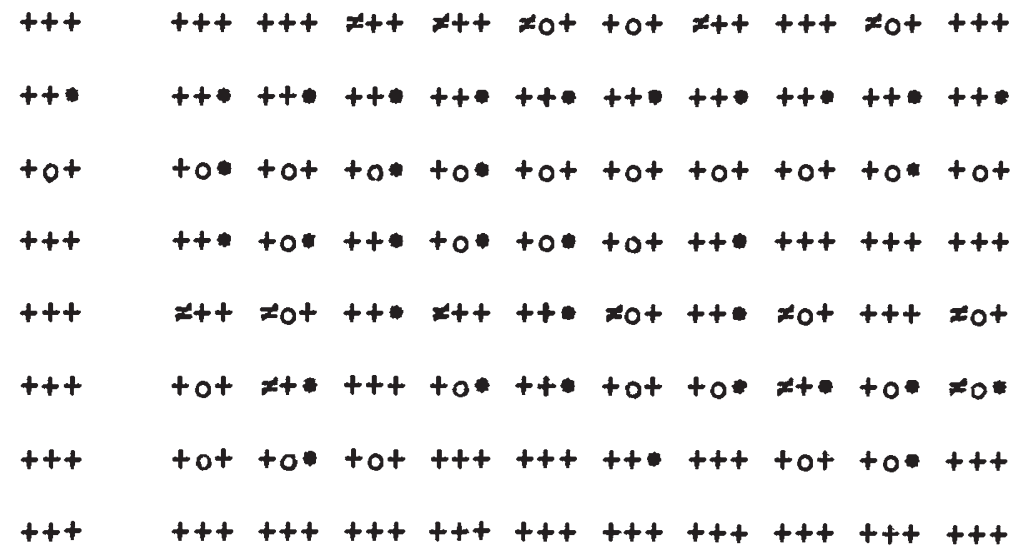

Fig. 7.-Facsimile of some of the test-cross families from the inbreeding-outbreeding experiments after 20 generations of outbreeding. Each line shows the phenotype of the sampled individual (isolated on the left) and those of the ten test-cross progeny derived from it. For example, the top line shows a parental genotype QqRrSS.

little improvement is possible with our present computer, except by unrealistic restrictions on the breeding system.

Three unlinked genes are involved, queer $(q)$, round $(r)$ and star $(s)$, with recessive phenotypes $\neq 0$ and $*$ respectively, the normal phenotypes being + in each case. There is no selection against $Q Q$, $Q q, R R, R r, S S$ or $S s$ (viability = I), but there is selection against $q q, r r$ and $s s$, which singly have viabilities of $\frac{3}{4}, \frac{7}{8}$ and $\frac{15}{16}$ respectively. For simplicity, there is no interaction between the genes, and the viability of a multiple recessive homozygote is the product of the appropriate single viabilities. Selection acts immediately after fertilisation, and eliminated individuals are never reckoned as part of the population, which consists entirely of breeding individuals; all surviving organisms have full reproductive capacity.

Selection is subject to chance, and it acts on each new individual in the following way. Each of the three genes is considered in turn. If it is homozygous recessive, then a random number is generated in the range o-3 for $q, 0-7$ for $r$ and o-I 5 for $s$. If zero, the individual is immediately discarded and the computer proceeds to form another; 
if not zero, the next gene is considered or if the process is now completed for all three genes, the individual goes into the new generation.

At the start of each run, whatever the breeding system, the gene frequencies are 50 per cent., and the genotypes are in precise HardyWeinberg proportions. This is an unnatural gene structure, but it allows very good illustration of the essential properties of the different breeding systems; in particular, the rapid increase in homozygosity

TABLE I

The numbers of different genotypes present in samples of 60 individuals taken after 20 generations from a fully inbreeding population, and after 20 and 60 generations from an outbreeding (panmictic) population.

The possible number of different genotypes is 27 . Normal phenotypes have their numbers in bold type. Selection against recessive homozygotes was greatest against $q q$, least against ss.

\begin{tabular}{|c|c|c|c|c|}
\hline \multirow{2}{*}{\multicolumn{2}{|c|}{ Generations }} & \multirow{2}{*}{$\begin{array}{c}\text { Inbreeding } \\
20\end{array}$} & \multicolumn{2}{|c|}{ Outbreeding } \\
\hline & & & 20 & 60 \\
\hline \multicolumn{5}{|c|}{ Completely homozygous : } \\
\hline QQRRSS & . & 45 & ro & 22 \\
\hline$\widetilde{Q} \widetilde{Q R R s S}$ & . & 10 & 5 & 3 \\
\hline QQ $r r S S$ & . & 2 & $\mathrm{I}$ & I \\
\hline$\widetilde{Q}$ Qrrss & . & 1 & $\ldots$ & ... \\
\hline$q q \bar{R} R S S$ & . & 2 & $\cdots$ & ... \\
\hline \multicolumn{5}{|c|}{ Heterozygous at I locus: } \\
\hline QQRRSs & .. & ... & 6 & 19 \\
\hline$\widetilde{Q Q R R S S}$ & . & $\ldots$ & 8 & 3 \\
\hline$\widetilde{Q} q R R S S$ & . & $\ldots$ & 4 & $\ldots$ \\
\hline$\widetilde{Q} Q R r s s$ & . & $\ldots$ & $I$ & 2 \\
\hline$\widetilde{Q}$ QrrSs & $\cdot$ & $\cdots$ & 2 & $\cdots$ \\
\hline \multicolumn{5}{|c|}{ Heterozygous at 2 loci : } \\
\hline QQRrSs & . . & $\cdots$ & 8 & 6 \\
\hline QqRRSs & . & $\ldots$ & 8 & 2 \\
\hline$\widetilde{Q} q R r S S$ & . & $\ldots$ & 2 & $\mathrm{I}$ \\
\hline$\widetilde{Q} q R r s s$ & . & $\cdots$ & 2 & $\cdots$ \\
\hline \multicolumn{5}{|c|}{ Heterozygous at all 3 loci : } \\
\hline$Q q R r S s$ & & $\cdots$. & 3 & $\mathrm{I}$ \\
\hline
\end{tabular}

on inbreeding is striking. There would be no point in beginning with a population near equilibrium - there would be little to happen.

With complete dominance in a population of living organisms, heterozygotes could not be recognised except by genetical tests. That situation is accepted in this experiment, so that the class may not only see the procedure which would have to be adopted in real life, but may also have an opportunity of participating.

The numbers of recessive homozygotes, which would normally be directly scored in the field, are shown on a monitoring tube, and to this extent the course of evolution can be followed. When it is desired to estimate the gene frequencies, heterozygosity and genetic variability 
of the population, a random sample of 60 individuals is taken and test-crossed against qqrrss. Each test-cross family is of ten individuals, and the print-out shows the phenotypes of the tested parent and the offspring. Fig. 7 shows a facsimile of part of such a print-out.

The 6o test-cross families are given to the class, who are required to score them and to deduce the genotypes of the sampled individuals. On this basis, the class can form an estimate of the genetic structure of the population. By temporarily sacrificing complete accuracy for speed, preliminary analyses of the population samples can be made

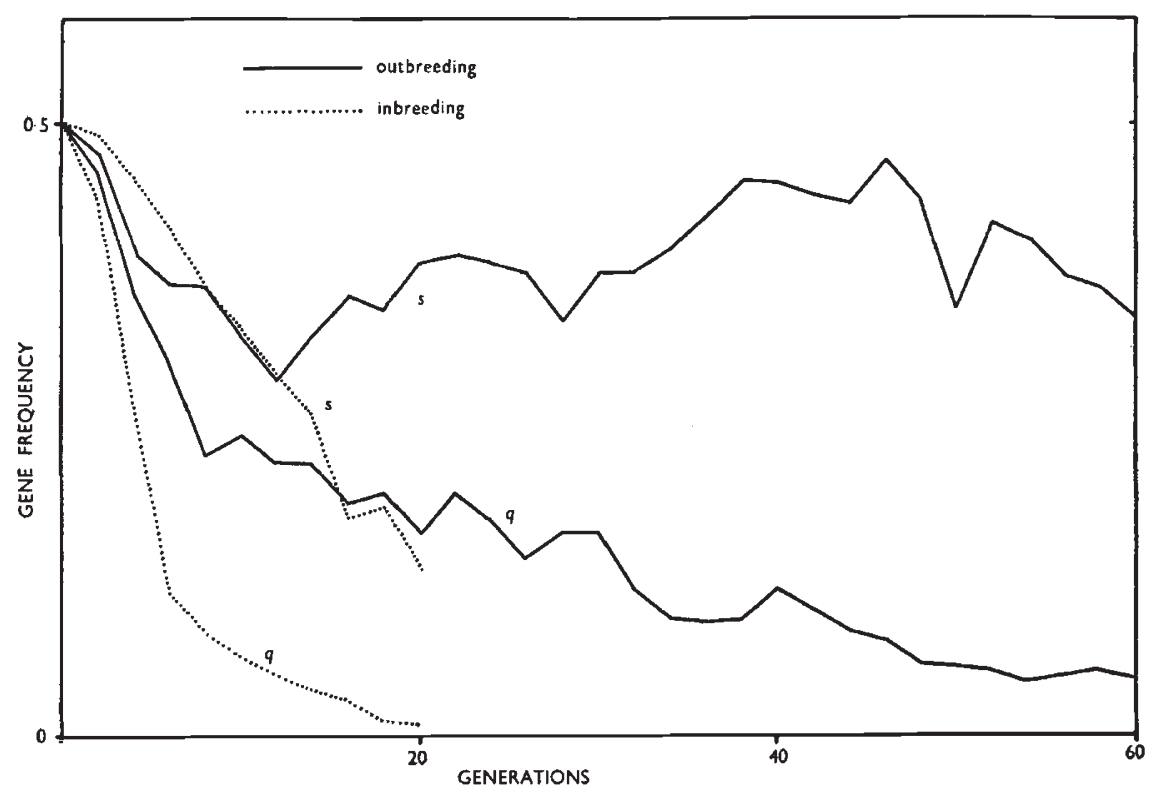

FIG. 8.-The progress of selection in inbreeding and in outbreeding (panmictic) populations, in actual teaching runs. Since all heterozygotes had long since disappeared from the inbreeding population by 20 generations, this run was discontinued then in order to leave more time for the outbreeding runs. Selection against recessive homozygotes only : relative viability of $q q$ is $\frac{s}{4}$, of $s s \frac{1}{1} \frac{5}{6}$. To avoid congestion, the curves for $r$ have been omitted.

as the experiment proceeds. As an example of the kind of picture which may be built up, table I shows results from two teaching runs in the same session, one inbreeding and one outbreeding, the former after 20 generations and the latter after 20 and after 60 generations.

It is possible to arrange for the computer to do some population analysis at regular intervals, and to print the results. These are not shown to the class until the experiment is completed, including the treatment of their own results. This further information allows the class to make a more detailed analysis of the progress of evolution, and in particular to see how the gene frequencies change. It also allows comparisons between samples and population. Fig. 8 relates to the same runs as table $\mathrm{I}$, and shows gene frequencies at intervals of two generations. Among other things, it illustrates rather well 
the greater importance of the Sewall Wright effect at lower selection pressures.

The test-crosses provide a statistical exercise as well as a genetical one, in showing variation on an expectation of $5: 5$. The occasional family of ten recessives from a phenotypically dominant parent draws attention to the possibility that the wrong conclusion may occasionally be drawn from a family of ten dominants ; this is why ten is preferred as the family size, rather than a more efficient higher number.

\section{DISCUSSION}

Although it is much too early for final assessment of the value of this new technique in genetics teaching, my experience so far is encouraging. There is certainly room for improvement, not only in the experiments themselves, but particularly in the method of presentation to the class. The experience of other teachers would be valuable, and I hope that this paper may encourage some of them to try this approach; I will gladly make my programme tapes available to departments which have access to a suitable computer. It is, I think, important that the students should be at the computer when the experiments are in progress. There is an important psychological difference between simply being handed results and actually being present as they are produced.

The experiments have been received with a mixture of curiosity and suspicion. Computers are still a novelty, and the opportunity of seeing one in action is eagerly accepted. There is thus initially a great interest in the experiments. In general, this seems to be maintained, and I think the classes have enjoyed the work and benefited from it. A common attitude to the evolution experiments has been "We've heard all about this in your lectures and read about it in books, but the significance didn't really sink in until we did these experiments and saw how striking the results were".

Suspicion that the results are faked is very natural, and an important aspect of presentation lies in its removal by making it clear that the teacher has no more control over the results than he has over those from experiments with living organisms. A typical comment is "But you can only get out of the machine what you put in ". An effective counter, quickly appreciated by the class, is "That is equally true of a Drosophila culture bottle". Different results when an experiment is repeated, and comparison with the results of previous classes, also help to convince the students that the experiments are genuine.

Not only do these experiments help to develop the student's imagination, but they provide a good basis on which to discuss the logical relationship between the "laws" of genetics, the experiments on which they are based, and the experiments commonly used to demonstrate them. There is a tendency among students to treat ordinary genetic experiments as "proving" Mendel's laws, and it is not always easy to make them realise that the production of a $3: \mathrm{I}$ 
ratio in a Drosophila experiment does no more than show that Drosophila gives the same results as Mendel's peas, and that if any other laws could explain Mendel's results, they would explain the class experiments too.

The quite different logical basis of computer experiments helps considerably in making this point, which is a most important one with honours students, for whom the assimilation of ideas is at least as valuable as learning the rules of genetics. Here, the laws of genetics are known, since they are predetermined in writing the programme; these experiments then demonstrate that given certain predetermined laws, the results tend to follow a particular pattern. For example, the explanation of Drosophila linkage results involves the assumption of various interferences in chiasma formation; the computer experiments show that if these interferences really do exist, then the results are very like those from Drosophila experiments. This may be emphasised by repeating the computer experiments with the interferences eliminated.

Care must be taken to avoid giving the impression that the computer experiments are valid imitations of Drosophila experiments. They do have complete validity, but it is only in respect of the genetics of certain special organisms-electronic ones.

The experiments which have been described are fairly simple ones designed to suit the genetics courses in Durham. They illustrate some of the basic principles of computer simulation, and clearly represent only a small fraction of the possible experiments. But the temptation must be avoided of introducing too much computer genetics, to the neglect of living organisms. Indeed, if computer models of genetic systems become an important part of a genetics course, it is especially necessary that proper use should be made of living organisms, or there will be a danger of the course becoming divorced from reality.

The elaboration of the experiments is another temptation to be guarded against, especially where easy accessibility of the computer removes one of the restraints operating at Durham. It is obvious that the evolution experiments are capable of considerable elaboration, but this is properly a matter for post-graduate work. While there is no doubt that computer techniques have a valuable potential in genetical research, that is beyond the scope of this paper. It is desirable for undergraduate teaching that the experiments should be few, simple, and to the point.

\section{SUMMARY}

An account is given of some experiments, based on computer simulation of genetic systems, which have been successfully used for practical work in genetics for undergraduate classes in the Durham Colleges. 
Accounts are given of visual demonstrations of meiosis, of chromosome mapping by linkage experiments, and of evolutionary experiments demonstrating the Sewall Wright effect and the differences. between inbreeding and outbreeding systems under selection.

Acknowledgments.-I would like to thank the Director of the Durham University Computing Laboratory (Dr E. S. Page) for the readiness with which the computer has been made available to us. My thanks are also due to the 1959-6o Final Honours classes in botany and zoology at Durham for their willing and intelligent cooperation during the early trials of these experiments.

\section{REFERENCES}

CROSBY, J. L. I96o. The use of electronic computation in the study of random fluctuations in rapidly evolving populations. Phil. Trans. Roy. Soc. B, 242, 55 I-573.

FRASER, A. S. 1957. Simulation of genetic systems by automatic digital computers. I. Introduction. Aust. 7. Biol. Sci., Io, 492-499.

FRASER, A. S. 1960. Simulation of genetic systems by automatic digital computers. VI. Epistasis. Aust. F. Biol. Sci., I3, I 50-162. 